\title{
Current-induced non-adiabatic spin torques and domain wall motion with spin relaxation in a ferromagnetic metallic wire
}

\author{
M. Thorwart and R. Egger \\ Institut für Theoretische Physik, Heinrich-Heine-Universität Düsseldorf, D-40225 Düsseldorf, Germany
}

(Dated: November 17, 2018)

\begin{abstract}
Within the $s$ - $d$ model description, we derive the current-driven spin torque in a ferromagnet, taking explicitly into account a spin-relaxing Caldeira-Leggett bath coupling to the $s$-electrons. We derive Bloch-Redfield equations of motion for the $s$-electron spin dynamics, and formulate a gradient expansion scheme to obtain non-adiabatic (higher-order) corrections to the well-known adiabatic (first-order) spin torque. We provide simple analytical expressions for the second-order spin torque. The theory is applied to current-driven domain wall motion. Second-order contributions imply a deformation of a transverse tail-to-tail domain wall. The wall center still moves with a constant velocity that now depends on the spin-polarized current in a non-trivial manner.
\end{abstract}

PACS numbers: 72.25.Ba, 72.25.Rb, 75.60.Ch

\section{INTRODUCTION}

Many recent experiments have brought to attention the intricate and rich interplay between spin-dependent transport and the magnetization dynamics in ferromagnetic (FM) materials [1, 2, 3]. A particularly interesting issue concerns the spin torque $\mathbf{T}[4,5,6,6,7,8,9]$ exerted by conduction electrons on the FM magnetization, $-M_{s} \mathbf{n}$, where $\mathbf{n}(x, t)$ is a space-time dependent unit vector and $M_{s}$ is assumed constant. The spin torque is crucial for the understanding of many topics of present scientific and technological interest, including current-driven domain wall (DW) motion [10], magnetization precession and switching in multilayer geometries [11], and spin transport in general [12]. Here, we study the spin torque exerted by a steady-state dc spin current $J_{s}$ [for the definition of $J_{s}$, see Eq. [10)] in the presence of spin relaxation. Common microscopic mechanisms responsible for spin relaxation are, for instance, provided by magnetic impurities or the various sources for spin-orbit scattering, but we shall focus on generic features and study a phenomenological Caldeira-Leggett-type heat bath describing random magnetic fields with Gaussian statistics which are responsible for spin relaxation.

As has been discussed recently, spin relaxation is essential in determining the non-adiabatic corrections to the well-known adiabatic spin torque [5, 6]

$$
\mathbf{T}_{a d}=-J_{s} \partial_{x} \mathbf{n} .
$$

It is common practice to call all terms beyond Eq. (11) 'non-adiabatic' (but see below). In particular, Zhang and Li [13] predicted a leading non-adiabatic spin torque contribution

$$
\mathbf{T}_{Z L}=\beta J_{s} \mathbf{n} \times \partial_{x} \mathbf{n},
$$

which was confirmed by later work [14, 15, 16, 17, 18], although some questions were raised in Ref. [19]. Here the dimensionless $\beta$ parameter is given as $\beta=\hbar / \Delta \tau_{s}$, where $\Delta$ is the FM exchange splitting and $\tau_{s}$ denotes a spin relaxation time. Although $\beta$ is generally small, $\mathbf{T}_{Z L}$ can profoundly influence many of the above-mentioned phenomena. Recently, a complicated and highly nonlocal form of the spin transfer torque including nonadiabatic corrections due to fast varying magnetic textures has been proposed [20]. This form includes indirect Ruderman-Kittel exchange interaction processes 21, 22] between the localized spins mediated by itinerant electrons. A scattering approach has been employed in Ref. 23. to calculate the spin torque in the limit of a sharp DW. However, it is difficult to deal with spin relaxation in that formalism. Strong enhancement of the spin torque and of the domain wall mobility due to spin-orbit coupling in magnetic II-V semiconductors has been reported in Ref. 24].

In this work, we provide a general derivation of the spin torque within the $s$ - $d$ model [25, 26], see Sec. [I] The selfconsistent Stoner model gives very similar results for this problem but is technically somewhat more demanding 15, 16, 18. While spin torques in the presence of spin relaxation have so far been studied mostly for small perturbations around a homogeneously magnetized ferromagnet [13, 15, 16], we here describe the theory for the leading nonadiabatic corrections for non-homogenous (both in space and time) magnetization profiles. For these corrections and not extremely large applied currents, the $s-d$ model is expected to provide a reasonable description. We note in passing that recently a simple classical model of non-adiabatic current-induced spin torques has been proposed [27]. In our work, spin relaxation is included within the weak-coupling Bloch-Redfield approach [28, 29, 30, 31], where the $s$-electrons are coupled to a spin-relaxing environment modelled by a phenomenological harmonic oscillator bath [32], see Sec. III. Moreover, the itinerant electron spins are influenced by a space-time dependent magnetic field produced by the localized electron spins. This external field also determines the stationary state to which the itinerant spins relax. Validity of the Bloch-Redfield approach requires weak coupling of the $s$-electrons to the bath, i.e., sufficiently long spin relaxation times $\tau_{s}$, and a bath memory time $\tau_{c}$ short compared to all other relevant timescales. 
Both conditions hold in most cases of practical interest.

Based on this formalism, we then determine the spin torque $\mathbf{T}$ acting on the FM magnetization $\mathbf{n}(x, t)$ within a gradient expansion around the adiabatic limit, see Sec. IV We illustrate the method by computing all firstand second-order derivative contributions to the spin torque, i.e., all terms that involve $\partial_{x, t} \mathbf{n}, \partial_{x, t}^{2} \mathbf{n}, \partial_{x} \partial_{t} \mathbf{n}$, or products of first-order derivatives. The first-order terms provide the leading terms in this expansion, which we call the adiabatic torque. Note that our convention disagrees with common usage but emphasizes that these terms completely dominate the spin torque for slowly varying $\mathbf{n}(x, t)$. Indeed, a first-order expansion recovers both Eq. (1) and (2). This shows that the 'non-adiabatic' term (2) is really a consequence of spin relaxation rather than being related to higher-order terms in the gradient expansion [13, 15, 16. . Our approach then allows to systematically evaluate higher orders in an expansion around the adiabatic limit. It should be stressed, however, that the $s$ - $d$ model may become difficult to justify for extremely sharp or fast features in $\mathbf{n}(x, t)$ (e.g. very narrow domain walls), and in practice this expansion is only useful for the first few orders.

Here we will focus in detail on the second-order spin torque contributions which turn out to be of relatively simple form. To illustrate some physical consequences of these new terms, in Sec. $\mathrm{V}$ we address the problem of DW motion driven by a spin-polarized current for the case of a tail-to-tail transverse DW. We find that the secondorder contribution leads to a deformation of the adiabatic DW shape. Nevertheless, under steady state conditions the DW center moves uniformly with constant terminal velocity $V_{D W}$. Importantly, this velocity depends nontrivially on the spin current density $J_{s}$. After following the known linear behavior of the velocity found from the first-order calculation for small $J_{s}, V_{D W}$ does not grow further with $J_{s}$ but instead starts to decrease. We expect that when including all orders, this would indicate saturation behavior. Our results seem in qualitative agreement with experimental observations, where measured DW velocities are typically smaller than those predicted by first-order calculations 33]. Moreover, the deformation of the DW profile is also observed in experiments and can even induce a transformation to a completely different DW type [34]. While the quantitative description of experiments on current-driven DW motion is likely to require more refined models, our principal aim is to provide general expressions for the leading corrections to the known spin torque expressions (11) and (2), and to illustrate typical effects caused by these new terms. The practical importance of such effects was also revealed by recent numerical studies [35, 36, 37.

Our paper then closes by offering some concluding remarks in Sec. VI. The derivation of the relaxation kernel under the Bloch-Redfield approach can be found in the Appendix. Throughout the paper, we put $\hbar=k_{B}=1$.

\section{MODEL}

We consider an (infinitely long) FM wire with homogeneous magnetization along the cross-section, $\mathbf{n}=\mathbf{n}(x, t)$, where $x$ is the longitudinal direction. This simplification allows us to work with an effectively one-dimensional (1D) theory. For thin FM nanowires with only a few occupied transverse sub-bands, this description is directly relevant [38], but for wider wires, nontrivial transversal magnetization profiles (such as vortex walls) are thereby excluded. Moreover, the 1D model also neglects spin waves propagating in the transverse direction that can be excited in wider wires. We note in passing that in strictly 1D metallic wires, electron-electron interactions can cause non-Fermi liquid effects [39] that could also influence domain-wall motion [40]. However, such effects are not studied in what follows.

Adopting the widely used $s-d$ model for itinerant FMs 2, 3, 25, 26], the relevant dynamical degrees of freedom are (i) the FM magnetization $\mathbf{n}(x, t)$ associated with localized $d$-electrons, and (ii) the conduction electron spin current density $\mathbf{J}(x, t)$ and spin density $\mathbf{s}(x, t)$, respectively, describing the delocalized $s$-electrons. The FM magnetization then obeys the Landau-Lifshitz-Gilbert (LLG) equation [2]

$$
\partial_{t} \mathbf{n}=-\gamma_{0} \mathbf{n} \times \mathbf{B}_{\mathrm{eff}}[\mathbf{n}]+\alpha \mathbf{n} \times \partial_{t} \mathbf{n}+\mathbf{T},
$$

where $\gamma_{0} \mathbf{B}_{\text {eff }}$ includes external, anisotropy, and exchange magnetic fields unrelated to the coupling to $s$ electrons. The Gilbert damping parameter $\alpha$ provides a phenomenological description of dissipative influences on $\mathbf{n}$ (again in the absence of $s$-electrons). Our principal aim is to find the spin torque $\mathbf{T}$ when the FM carries a (spin-polarized) current and both spin relaxation and magnetic texture (spatio-temporal variations of $\mathbf{n}$ ) are present.

Let us then address the conduction electron degrees of freedom. Expressed in terms of right- and left-moving $(p=R / L)$ quasi-particle annihilation operators $c_{p, \sigma}(x)$ for spin $\sigma=\uparrow, \downarrow$, the 1D spin and spin current density are defined as

$$
\mathbf{s}(x)=\mathbf{J}_{R}(x)+\mathbf{J}_{L}(x), \quad \mathbf{J}(x)=v\left(\mathbf{J}_{R}(x)-\mathbf{J}_{L}(x)\right),
$$

where $v$ is the spin velocity and the chiral currents are

$$
\mathbf{J}_{R / L}(x)=\frac{1}{2}: c_{R / L}^{\dagger}(x) \vec{\sigma} c_{R / L}(x): \text {. }
$$

Here colons denote normal ordering, spin indices are left implicit, and $\vec{\sigma}$ are standard Pauli matrices acting in spin space. The currents (5) obey the $S U(2)$ Kac-Moody algebra [39]. Within this 1D description, the low-energy Hamiltonian describing the conduction electrons is universal and given by

$$
H_{0}=-i v \sum_{p=R / L= \pm} p \int d x c_{p}^{\dagger} \partial_{x} c_{p} .
$$


The spin sector of $H_{0}$ can now be expressed directly in terms of the currents (5), and completely decouples from the charge sector. (In a strictly 1D wire, this procedure works even when forward-scattering electron-electron interactions are included [39].) The resulting Sugawara representation of the spin part of $H_{0}$ (the charge part is irrelevant and omitted here) is

$$
H_{0}=\frac{v}{4} \int d x: \mathbf{s} \cdot \mathbf{s}+\frac{1}{v^{2}} \mathbf{J} \cdot \mathbf{J}: \text {. }
$$

Within the $s$ - $d$ model, $s$-electrons are coupled to the time-dependent magnetization $\mathbf{n}(x, t)$ only by exchange processes. Effectively, the magnetization then acts on the $s$-electrons as a time-dependent external field,

$$
H_{e x}(t)=\Delta \int d x \mathbf{s}(x) \cdot \mathbf{n}(x, t),
$$

where $\Delta$ is proportional to the FM exchange coupling and can have either sign. For the resulting 'system' (the $s$-electrons), we then arrive at the Hamiltonian $H_{S}(t)=$ $H_{0}+H_{e x}(t)$. Equation (8) now generates a spin torque entering Eq. (3),

$$
\mathbf{T}=\mathbf{n} \times\left(-\frac{\delta H_{e x}}{\delta \mathbf{n}}\right)=-\Delta \mathbf{n} \times \mathbf{s} .
$$

Our task is then to compute the spin density $\mathbf{s}(x, t)$ in the presence of an external dc spin current $J_{s}$ and for a given dynamical magnetization profile $\mathbf{n}(x, t)$. We mention in passing that we do not perform a unitary transformation into an adiabatic reference frame where the local quantization axis for the itinerant electrons is aligned with $\mathbf{n}(x, t)$. Albeit this transformation has been used in many papers on the subject, see e.g. Ref. 41] and references therein, we believe that it does not offer advantages in the presence of spin relaxation.

In order to enforce a finite spin current, in principle (i) we need to resort to a non-equilibrium formalism. In addition, (ii) we need a proper description of the relaxation processes driving the momentary state to a stationary state. Let us first discuss issue (i), where a simple boundary condition can be used instead of the full dynamical formalism, see also Ref. [38]. We want to describe a steady-state situation with externally imposed constant spin current

$$
J_{s}=\frac{P I}{e A},
$$

expressed in terms of the charge current $I$ flowing through a wire of cross-section $A$ with spin polarization $0<P \leq 1$. Since this stationary spin current is externally enforced, we include it as a boundary condition in the equation of motion of the itinerant electrons derived below. This boundary condition has to be imposed far away from any magnetic texture (i.e., at $x \rightarrow \mp \infty)$, where it implies a constant spin current $\mathbf{J}=J_{s} \mathbf{n}$. Only around, say, a DW center, the true spin current density deviates from $\mathbf{J}(x, t)=J_{s} \mathbf{n}(x, t)$. Coming to issue (ii), it is then tempting to identify the steady state to which an actual spin current configuration tends to relax with $\mathbf{J}(x, t)=J_{s} \mathbf{n}(x, t)$. This indeed we find, see Sec. III] The Heisenberg equations of motion, $\partial_{t} \mathbf{J}_{R / L}=i\left[H_{S}(t), \mathbf{J}_{R / L}\right]$, now yield operator equations for the spin density and the spin current,

$$
\begin{aligned}
\partial_{t} \mathbf{s}+\partial_{x} \mathbf{J} & =-\Delta \mathbf{s} \times \mathbf{n}+\boldsymbol{\Gamma}_{s}, \\
\partial_{t} \mathbf{J}+v^{2} \partial_{x} \mathbf{s} & =-\Delta \mathbf{J} \times \mathbf{n}+\boldsymbol{\Gamma}_{J} .
\end{aligned}
$$

The relaxation terms $\boldsymbol{\Gamma}_{s / J}[\mathbf{s}, \mathbf{J}]$ due to the coupling of the $s$-electrons to the spin-relaxing environment are specified in Sec. III. Far away from magnetic textures, both the derivative terms and the relaxation terms $\boldsymbol{\Gamma}_{s, J}$ are irrelevant, and taking $\mathbf{s}=s_{0} \mathbf{n}$ and $\mathbf{J}=J_{0} \mathbf{n}$ with arbitrary constant coefficients $s_{0}$ and $J_{0}$ solves these equations. The boundary condition on $\mathbf{J}$ discussed above then enforces $J_{0}=J_{s}$. To determine $s_{0}$, one has to find the stationary state of the equation of motion (11) in the presence of spin relaxation. We here anticipate the result $s_{0}=-\chi_{s} \Delta$ derived in Sec. III, see Eq. (16), where $\chi_{s}=(2 \pi v)^{-1}$ is the spin susceptibility.

To summarize our discussion, we seek solutions to Eqs. (11) and (12) of the form

$$
\begin{aligned}
& \mathbf{J}(x, t)=J_{s} \mathbf{n}(x, t)+\sum_{k=1}^{\infty} \mathbf{J}_{k}(x, t), \\
& \mathbf{s}(x, t)=-\left(\chi_{s} \Delta\right) \mathbf{n}(x, t)+\sum_{k=1}^{\infty} \mathbf{s}_{k}(x, t),
\end{aligned}
$$

where the $k$ th-order terms contain $k$ th-order spatiotemporal derivatives of $\mathbf{n}$, or respective products of lowerorder derivatives. Such an expansion provides a systematic way to classify deviations from the adiabatic limit where only the $k=1$ terms are retained. However, as we have discussed above, in practice only the first few orders in this expansion can be reliably extracted under the $s$ - $d$ model description.

\section{SPIN RELAXATION}

For a description of the spin-relaxing environment acting on the spin dynamics of the $s$-electrons, we employ a Caldeira-Leggett system-bath approach [32], where the $s$-electron spin density and spin current are linearly coupled to fluctuating magnetic fields $\mathbf{B}_{s, J}(x)$,

$$
H_{S B}=\int d x\left[\mathbf{B}_{s}(x, t) \cdot \mathbf{s}(x)+\mathbf{B}_{J}(x, t) \cdot \mathbf{J}(x)\right] .
$$

These fields provide a simple phenomenological modelling of the effects of random magnetic impurities and of spin-orbit scattering. The Ansatz (15) assumes 'soft' forward scattering due to the fluctuating fields, where rightand left-moving fermions retain their chirality. Backward scattering processes, for instance associated with 
elastic potential scattering [38], will modify our quantitative conclusions, as discussed in more detail below. Note also that the fluctuating fields are assumed to solely couple to $s$-electrons, and the FM magnetization $\mathbf{n}(x, t)$ is only affected indirectly via its exchange coupling to the $s$-electron spin density $\mathbf{s}(x, t)$. The Hamiltonian $H_{B}$ describing the uncoupled fields $\mathbf{B}_{s, J}$ corresponds to a suitable bath of harmonic oscillators [32. With $\overline{\left\langle\mathbf{B}_{s, J}\right\rangle}=0$, where $\overline{\langle O\rangle}$ denotes the equilibrium quantum-statistical average of some operator $O$ with respect to $H_{B}$, all relevant properties are thus encoded by specifying their two-point correlation functions. The total Hamiltonian is then $H_{t o t}(t)=H_{S}(t)+H_{S B}+H_{B}$, with $H_{S}(t)=H_{0}+H_{e x}(t)$ as given in Eqs. (7) and (8).

To describe the effects of Eq. (15) on the $s$-electron spin dynamics, we adapt the textbook weak-coupling Bloch-Redfield approach [28, 29, 30, 31] to a field theory as required here. For the convenience of the interested reader, we provide a detailed discussion of the BlochRedfield equations in the Appendix. The basic assumptions underlying this approach are (i) short bath correlation times, i.e., the two-point correlation functions of $\mathbf{B}_{s, J}(x, t)$ vanish on a timescale $\tau_{c}$ small compared to all relevant other timescales, and (ii) weak system-bath coupling $H_{S B}$. Note that $H_{S B}$ in Eq. (15) is of the general form considered in the Appendix, see Eq. (A1). This implicitly also requires that we stay not too far away from the adiabatic limit. Following the steps in the Appendix, we obtain the relaxation term entering Eq. (11) in the form

$$
\boldsymbol{\Gamma}_{s}=-\frac{\mathbf{s}(x, t)+\left(\chi_{s} \Delta\right) \mathbf{n}(x, t)}{\tau_{s}},
$$

where $\chi_{s}=(2 \pi v)^{-1}$ and $\tau_{s}$ is the spin relaxation time. Here $\tau_{s}$ is assumed uniform in the $x, y, z$ directions; the generalization is discussed in the Appendix. Since $\mathbf{n}(x, t)$ acts effectively as a space-time dependent magnetic field on the spin density s, see Eq. (8), it directly appears in the stationary state approached in the relaxation term (16). In a similar manner, we obtain

$$
\boldsymbol{\Gamma}_{J}=-\frac{\mathbf{J}(x, t)-J_{s} \mathbf{n}(x, t)}{\tau_{J}} .
$$

For the simple system-bath coupling (15), which disregards backscattering processes, the relaxation times $\tau_{s}$ and $\tau_{J}$ turn out to be equal. In the following, we shall allow for different relaxation times $\tau_{J} \neq \tau_{s}$, which results when allowing for more general models involving backscattering processes [38], i.e., when elastic disorder is present.

Before discussing the resulting spin torque, let us briefly comment on the validity regime of our description. Both the derivation of the relaxation terms, see Eqs. (16) and (17), and the iterative procedure for solving the resulting equations, see Sec. IV suppose that we are not too far away from the adiabatic limit. By that we mean that the space (time) variation of the magnetization direction $\mathbf{n}(x, t)$ is sufficiently smooth (slow) when compared to a characteristic lengthscale $\lambda$ (time scale $\lambda / v)$. This 'spin transport lengthscale' has been estimated by Zhang and Li, see Ref. [13]. Quantitatively, we suppose that both dimensionless non-negative functions $\lambda\left|\partial_{x} \mathbf{n}(x, t)\right| \ll 1$ and $(\lambda / v)\left|\partial_{t} \mathbf{n}(x, t)\right| \ll 1$. Under these conditions, an iterative expression like Eq. (18) implies a well-behaved perturbation series, where higher and higher orders give smaller and smaller corrections.

\section{SPIN TORQUE}

We next discuss how to extract the spin torque from an iterative solution of Eqs. (11) and (12). To that end, we perform a gradient expansion indicated by the series in Eqs. (13) and (14). This gradient expansion then generates a series for the spin torque,

$$
\mathbf{T}(x, t)=\sum_{k=1}^{\infty} \mathbf{T}_{k}(x, t) .
$$

Of course, the zero-order terms in Eqs. (13) and (14) do not generate a torque. This expansion naturally suggests to define the adiabatic spin torque as $\mathbf{T}_{1}$, the dominant piece for sufficiently slow and smooth variation of $\mathbf{n}(x, t)$. As remarked above, this definition differs from standard usage but provides a systematic way to classify different spin torque contributions.

\section{A. Adiabatic spin torque}

Let us now compute the first-order spin torque $\mathbf{T}_{1}$. The derivatives of $\mathbf{s}_{1}$ or $\mathbf{J}_{1}$ are of second order and can be dropped here. Inserting the Ansatz (13) and (14) into Eqs. (11) and (12) then gives

$$
\begin{aligned}
\left(\tau_{s}^{-1}-\Delta \mathbf{n} \times\right) \mathbf{s}_{1} & =\chi_{s} \Delta \partial_{t} \mathbf{n}-J_{s} \partial_{x} \mathbf{n} \equiv \mathbf{a}_{1} \\
\left(\tau_{J}^{-1}-\Delta \mathbf{n} \times\right) \mathbf{J}_{1} & =-J_{s} \partial_{t} \mathbf{n}+v^{2} \chi_{s} \Delta \partial_{x} \mathbf{n} \equiv \mathbf{b}_{1}
\end{aligned}
$$

Here $\mathbf{J}_{1}$ and $\mathbf{s}_{1}$ completely decouple. Exploiting that the equation $\lambda \mathbf{x}+\mathbf{y} \times \mathbf{x}=\mathbf{v}$ with real $\lambda$ and real 3 -vectors $\mathbf{x}$ and $\mathbf{y}$ is solved by

$$
\mathbf{x}=\frac{\lambda^{2} \mathbf{v}+\lambda \mathbf{v} \times \mathbf{y}+(\mathbf{v} \cdot \mathbf{y}) \mathbf{y}}{\lambda\left(\lambda^{2}+|\mathbf{y}|^{2}\right)},
$$

it is straightforward to obtain

$$
\begin{aligned}
\Delta \mathbf{s}_{1} & =-\mathbf{a}_{1} \times \mathbf{n}+\beta \mathbf{a}_{1}, \\
\Delta \mathbf{J}_{1} & =-\mathbf{b}_{1} \times \mathbf{n}+\tilde{\beta} \mathbf{b}_{1},
\end{aligned}
$$

where we have introduced the dimensionless parameters

$$
\beta=\hbar /\left(\Delta \tau_{s}\right), \quad \tilde{\beta}=\hbar /\left(\Delta \tau_{J}\right)
$$

In accordance with the weak-coupling Bloch-Redfield approach, in numerical prefactors we have neglected terms 
of order $\beta^{2}$ and $\tilde{\beta}^{2}$ against unity. The parameters $\beta$ and $\tilde{\beta}$ are small expansion parameters in this approach.

For the discussion of the adiabatic spin torque $\mathbf{T}_{1}$, we only need $\mathbf{s}_{1}$ as given in Eq. (21). Inserting this into Eq. (9) gives the complete first-order spin torque,

$$
\mathbf{T}_{1}=-J_{s} \partial_{x} \mathbf{n}+\beta J_{s} \mathbf{n} \times \partial_{x} \mathbf{n}+\Delta \chi_{s}\left[\partial_{t} \mathbf{n}-\alpha^{\prime} \mathbf{n} \times \partial_{t} \mathbf{n}\right],
$$

where $\alpha^{\prime}=\beta$. The first term is the adiabatic spin torque (1), and the second term is the 'non-adiabatic' correction (2). Moreover, the third term can be absorbed by renormalizing the gyromagnetic ratio $\gamma_{0}$ and related parameters, cf. Ref. [13]. Finally, the last term gives an additional contribution to the Gilbert damping that is absorbed by a redefinition of $\alpha$. Our assumption of equal longitudinal and transverse relaxation times, $\tau_{x, y}=\tau_{z}=\tau_{s}$, cf. Appendix, strictly implies that $\alpha^{\prime}=\beta$. Under more general conditions, however, this relation will not hold anymore [16, 18]. The approach presented here can straightforwardly be generalized in this direction. Equation (24) thus confirms and reproduces the results of Zhang and Li [13], and effectively yields the first-order (adiabatic) spin torque in the form

$$
\mathbf{T}_{1}=\mathbf{T}_{a d}+\mathbf{T}_{Z L}
$$

\section{B. Non-adiabatic spin torque}

Next we calculate the second-order terms in Eq. (18). The second-order contributions $\mathbf{s}_{2}$ and $\mathbf{J}_{2}$ are obtained by inserting the Ansatz (13) and (14) into Eqs. (11) and (12), thereby exploiting Eq. (19). Dropping terms of higher than second order, the resulting equations can be solved again, with the result

$$
\begin{aligned}
\Delta \mathbf{s}_{2} & =\mathbf{a}_{2} \times \mathbf{n}-\beta \mathbf{a}_{2}, \\
\Delta \mathbf{J}_{2} & =\mathbf{b}_{2} \times \mathbf{n}-\tilde{\beta} \mathbf{b}_{2},
\end{aligned}
$$

with the auxiliary vectors

$$
\begin{aligned}
& \mathbf{a}_{2}=\partial_{t} \mathbf{s}_{1}+\partial_{x} \mathbf{J}_{1}, \\
& \mathbf{b}_{2}=\partial_{t} \mathbf{J}_{1}+\partial_{x} \mathbf{s}_{1},
\end{aligned}
$$

where again terms of order $\beta^{2}$ were discarded against unity in prefactors. We have also omitted terms $\propto \mathbf{n}$ resulting from Eq. (20) since these do not generate a spin torque. In fact, only $\mathbf{s}_{2}$ in Eq. (26) is required. After straightforward algebra, we get

$$
\begin{aligned}
\mathbf{T}_{2} & =\mathbf{n} \times\left(c_{t x} \partial_{t} \partial_{x} \mathbf{n}+c_{t t} \partial_{t}^{2} \mathbf{n}+c_{x x} \partial_{x}^{2} \mathbf{n}\right. \\
& \left.+\mathbf{n} \times\left[d_{t x} \partial_{t} \partial_{x} \mathbf{n}+d_{t t} \partial_{t}^{2} \mathbf{n}+d_{x x} \partial_{x}^{2} \mathbf{n}\right]\right)
\end{aligned}
$$

with the coefficients

$$
\begin{aligned}
& c_{t x}=2 J_{s} / \Delta, \quad c_{t t}=c_{x x} / v^{2}=-\chi_{s}, \\
& d_{t x}=-(3 \beta+\tilde{\beta}) J_{s} / \Delta, \\
& d_{t t}=2 \beta \chi_{s}, \quad d_{x x}=v^{2} \chi_{s}(\beta+\tilde{\beta}) .
\end{aligned}
$$

We stress that as long as the magnitude of the magnetization vector is conserved, $\mathbf{n}^{2}(x, t)=1$, Eq. (30) represents the most general second-order spin torque allowed by symmetry constraints. At this stage, let us compare to the results of Ref. [15], where a spin torque contribution $\mathbf{T}_{2}^{\prime}=\left(J_{s} / \Delta\right) \mathbf{n} \times \partial_{t} \partial_{x} \mathbf{n}$ was reported. This should match our $c_{t x}$-term, but differs by a factor 2 . The reason for this difference is explained by noting that Ref. [15] employed the self-consistent Stoner description, where one finds indeed a factor 2 difference to our $s$ - $d$ model prediction [42]. The other contributions to the second-order torque in Eq. (30), in particular the $d_{t x}$-term, have not been reported before. Interestingly, the spin torque contains $J_{s}$-independent and linear-in- $J_{s}$ terms only. However, even the linear-in- $J_{s}$ spin torque (30) leads to a nonlinear response of the DW velocity as a function of $J_{s}$, see Sec. V.

The second order torque $\mathbf{T}_{2}$ in Eq. (30) introduces several new features into the LLG equation (3). First, the only terms proportional to the spin current $J_{s}$ are due to $c_{t x}$ and $d_{t x}$. All other terms are less important when large spin currents are applied. For instance, the $c_{x x^{-}}$ term can be combined with the exchange term in the effective magnetic field $\mathbf{B}_{\text {eff }}$ entering the LLG equation (3), see below in Eq. (33), i.e., it can be absorbed by a renormalization of the exchange constant $J$. Moreover, the $c_{t t}$ and $d_{t t}$-terms introduce acceleration terms in the LLG equation. Since we are interested in current-driven spin torques here, we will exclusively focus on the $c_{t x}$ and $d_{t x}$ terms from now on. We have checked explicitly that the results below do not qualitatively change if the $d_{x x}, c_{t t}, d_{t t}$ terms are included in a perturbative scheme. The second-order spin torque is thus taken as

$$
\mathbf{T}_{2}=\frac{2 J_{s}}{\Delta} \mathbf{n} \times\left(\partial_{t} \partial_{x} \mathbf{n}-\frac{3 \beta+\tilde{\beta}}{2} \mathbf{n} \times \partial_{t} \partial_{x} \mathbf{n}\right)
$$

This is a central result of our work. Albeit the coefficient $d_{t x}$ is smaller by a factor $\approx 2 \beta$ compared to $c_{t x}$, it can qualitatively influence the magnetization dynamics, just as is the case for the first-order terms. We believe that Eq. (32) should be included in micromagnetic simulations of (for instance) DW dynamics. Below we will see that these new terms lead, among other phenomena, to a deformation of the shape of a DW, as the spin torque now involves the time derivative of the gradient of $\mathbf{n}$.

\section{DOMAIN WALL MOTION}

In order to illustrate the effects of the new spin torque terms (32), we now consider the problem of currentinduced domain wall motion. Let us take a tail-to-tail DW, being a solution of the stationary LLG equation (3) with

$$
\gamma_{0} \mathbf{B}_{\mathrm{eff}}[\mathbf{n}]=J \partial_{x}^{2} \mathbf{n}+K n_{z} \hat{e}_{z}-K_{\perp} n_{y} \hat{e}_{y},
$$

where $J$ is the ferromagnetic exchange coupling, $K$ the longitudinal and $K_{\perp}$ the transverse anisotropy constant 
(incorporating the effects of demagnetizing fields). The resulting initial DW configuration at time $t=0$ (when the spin current is switched on) with arbitrary center $x_{0}$ is then given by

$$
\mathbf{n}(x, 0)=\cosh ^{-1}\left(\frac{x-x_{0}}{w}\right) \hat{e}_{x}+\tanh \left(\frac{x-x_{0}}{w}\right) \hat{e}_{z},
$$

where $w=\sqrt{J / K}$ is the initial width of the wall. We then determine the time evolution of this configuration from the LLG equation in the presence of a spin polarized current $J_{s}$ switched on at $t=0$, taking the full spin torque $\mathbf{T}=\mathbf{T}_{1}+\mathbf{T}_{2}$, see Eqs. (25) and (32).

Due to the complexity of the resulting LLG equation, we have to solve it numerically. To that end, we spatially discretize the LLG equation into a discrete 1D spin chain, with spins $\mathbf{n}_{i}$ at $x=i a$ (integer $i$ ) for lattice constant $a$. The resulting first-order ordinary differential equation (in time) is then solved numerically starting from the initial configuration (34). To be specific, we choose a spatial grid with $x \in[0,1000 a]$ and scale time in units of $t_{0}=a / v$ (and energies correspondingly). In these units, we take [37] $J=3, K=$ $0.1, K_{\perp}=0.01, \Delta=0.2$, and $x_{0}=500 a$. This implies the initial DW width $w \simeq 5.5 a$. Moreover, we choose $\alpha=\beta=\tilde{\beta}=0.03$. Let us briefly estimate the corresponding dimensionful parameters. For this, we take the Fermi velocity of permalloy, $v=2.2 \times 10^{5} \mathrm{~m} / \mathrm{s}$ [43], with a unit cell of volume $V_{0}=a^{3}=(1 \mathrm{~nm})^{3}$. The time scale $t_{0}=4.5 \times 10^{-15} \mathrm{~s}$ follows. The values for the magnetic constants are $J=4.4 \times 10^{-10} \mathrm{~J} / \mathrm{m}, K=14.5 \times 10^{6} \mathrm{~J} / \mathrm{m}^{3}$, $K_{\perp}=14.5 \times 10^{5} \mathrm{~J} / \mathrm{m}^{3}$, and $\Delta=0.18 \mathrm{eV}$, resulting in the spin-flip time $\tau_{s}=1 /(\Delta \beta)=7.7 \times 10^{-13} \mathrm{~s}$. Note that the spin flip length for permalloy is $l_{s} \simeq 5 \mathrm{~nm}$ [44] corresponding to a spin flip time of $\tau_{s}=2.3 \times 10^{-14} \mathrm{~s}$. A spin current density of $J_{s}=0.2$ then corresponds to a charge current density (with polarization $P=1$ ) of $I / A=7 \times 10^{12} \mathrm{~A} / \mathrm{m}^{2}$. Typical current densities realized in experiments are of this order of magnitude [33, 34].

The numerical results for the DW configuration at times $t=80 t_{0}$ and $t=110 t_{0}$ for $J_{s}=0.1$ are shown in Fig. 1. As can be seen, the initial DW is moved to the right due to the presence of the spin current. Importantly, the second-order spin torque leads to a considerable deformation of the initial shape of the DW, where the magnetization rotates into the $x-y$ plane around the center of the DW. This region grows with time, but the DW center still moves along the direction of the current. For comparison, we also depict the results when only the first-order spin-torque is taken into account. This shows that the smearing of the DW profile is caused by the second-order torque. The strong DW deformation is the reason why a simple Walker Ansatz [6, 8, 13, 45] does not work. In the Walker Ansatz, one takes an adiabatic DW shape whose center is moving with some velocity $V_{D W}(t)$, a spatially independent out-of-plane angle $\phi(t)$, and a trial function for the polar angle $\theta\left(\left[x-\int_{0}^{t} d \tau V_{D W}(\tau)\right] / w(t)\right)$ with time-dependent

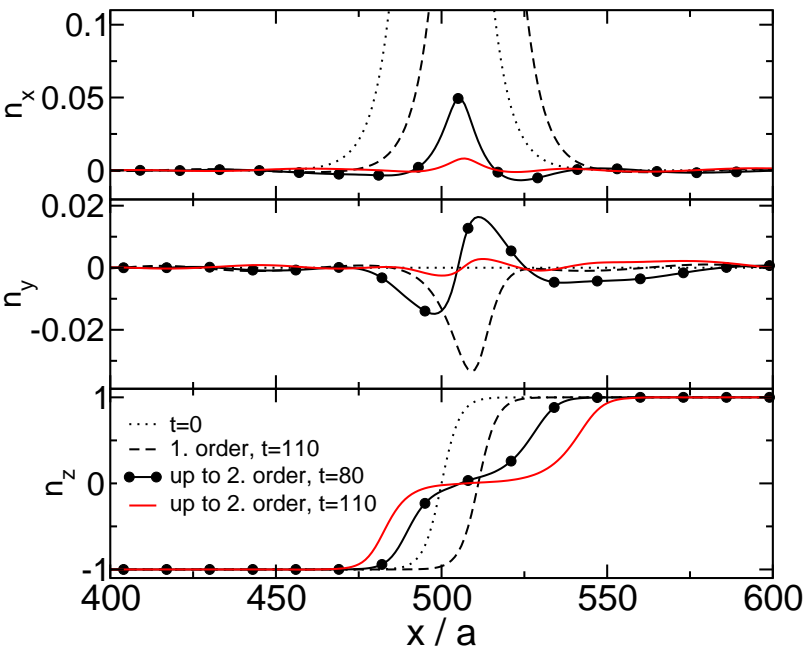

FIG. 1: (Color online) DW configuration at times $t=80 t_{0}$ [black solid line with filled circles] and $t=110 t_{0}$ [red solid line] for $J_{s}=0.1$ starting from the initial DW configuration (34) [dotted line]. For comparison, the result of keeping only $\mathbf{T}_{1}$ is also shown (for $t=110 t_{0}$, dashed line).

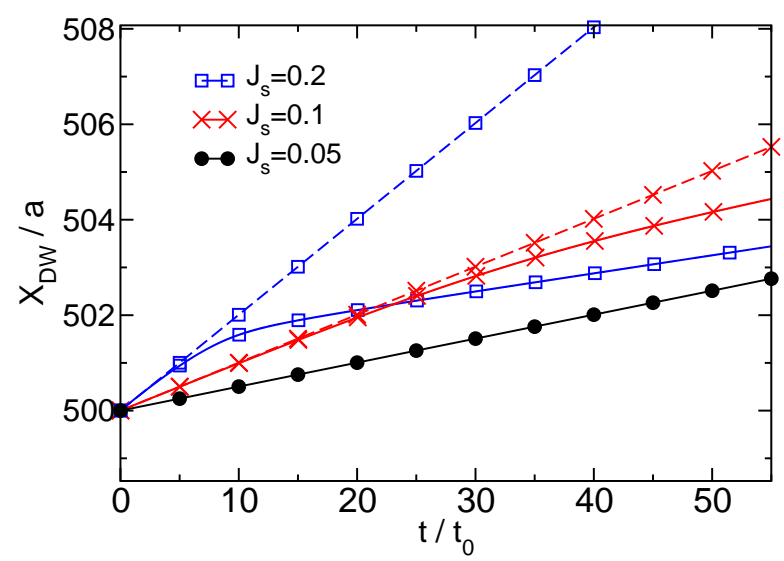

FIG. 2: (Color online) Time dependence of DW center for the same parameters as in Fig. 1] for $J_{s}=0.05(\bullet), 0.1(\times)$ and $0.2(\square)$. Shown are the results with the spin torque included up to first-order (dashed lines) and up to second order (full lines). For $J_{s}=0.05$, both coincide in the shown regime of time (black lines and filled circles).

DW width $w(t)$. Inserting such an Ansatz into the full LLG equations yields four coupled differential equations for three variables $\left(\phi(t), V_{D W}(t)\right.$ and $\left.w(t)\right)$. Unfortunately, there is thus no consistent solution under a Walker Ansatz.

Despite the deformation of the DW, the DW center $X_{D W}$ (defined as the zero of the $n_{z}$-component) displays a uniform steady-state translational motion. This is shown in Fig. 2 where $X_{D W}(t)$ is shown for different $J_{s}$. For the smallest value of the spin current density, $J_{s}=0.05$, the first- and second-order results for $X_{D W}(t)$ coincide in the shown regime of time. For larger spin currents, however, the DW center initially moves mainly due 


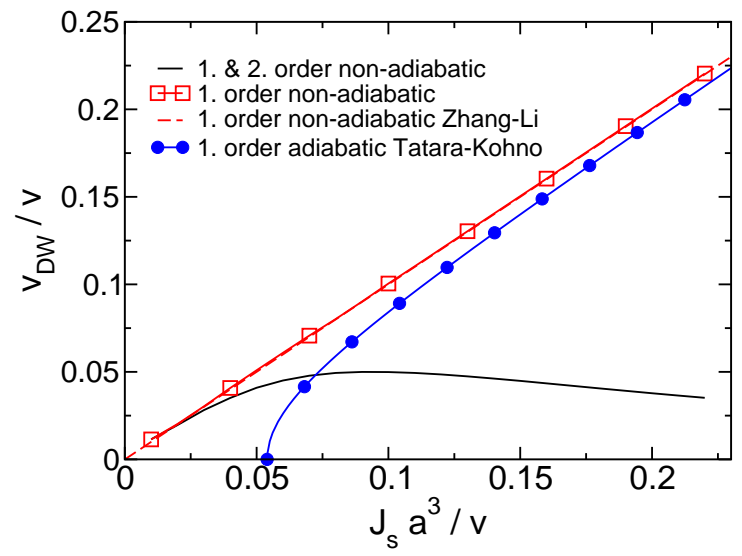

FIG. 3: (Color online) DW velocity vs. spin current density $J_{s}$ for same parameters as in Fig. 1 The black solid line gives the full numerical result, red empty squares the one when keeping only the first-order spin torque. The red dashed line gives the analytical result of Ref. [13] (for the full first-order spin torque), the blue full line with filled circles gives the result of Ref. [8] where only the spin torque (1) is kept.

to the first-order torque, and only after a transient time the DW is deformed. The motion of the DW center is then slower but still uniform, and a constant DW velocity can be extracted. At long times $V_{D W}(t)=d X_{D W}(t) / d t$ approaches the constant value $V_{D W}$, shown in Fig. 3 as a function of $J_{s}$. We also show the DW velocity following from only the first-order torque, where our numerical results reproduce the known result $V_{D W}=\beta J_{s} / \alpha$ [13]. As one can see, for small $J_{s}$, the first- and second-order results merge. However, for growing $J_{s}$, while the adiabatic (first-order) calculation gives a steadily growing DW velocity with growing $J_{s}$, the second-order (non-adiabatic) calculation indicates a non-monotonic dependence. This is a central result of our work, as it clearly shows that a larger spin current does not necessarily increase the DW velocity. Instead, it may lead to a DW deformation and a slower DW steady motion. This finding seems in qualitative agreement with experimental observations where the measured DW velocities are typically smaller than those predicted by the so far available first-order calculations [33]. We note that deviations from the first-order result become noticeable around $J_{s} \simeq 0.05$ which corresponds to a charge current density of $I / A=1.75 \times 10^{12} \mathrm{~A} / \mathrm{m}^{2}$ for our parameters (with $P=1$ ). This value should be compared, for instance, with the applied current densities of $I / A=1.0 \times 10^{12} \mathrm{~A} / \mathrm{m}^{2}$ in Ref. 33 and of $I / A=2.2 \times 10^{12}$ $\mathrm{A} / \mathrm{m}^{2}$ in Ref. 34 , indicating that the contribution of the second-order spin torque can be significant already for intermediate realistic current densities.

In Fig. 3, we also show the result of Ref. [8] where only the spin torque (1) was included, leading to $V_{D W} \simeq$ $\sqrt{J_{s}^{2}-J_{s, c r}^{2}}$ with the critical threshold spin current $J_{s, c r}=K_{\perp} w$. The DW would then be pinned by the transverse anisotropy for small $J_{s}$. However, as has been discussed in Ref. [13], the full first-order spin torque lifts this pinning.

Finally, we note that our formalism allows to numerically solve the three coupled equations of motion for $\mathbf{n}(x, t), \mathbf{s}(x, t)$ and $\mathbf{J}(x, t)$ directly. In particular, Eqs. (3), (11) and (12), together with Eqs. (16) and (17), with the initial configurations given in Eq. (34) for $\mathbf{n}(x, 0)$ and $\mathbf{s}(x, 0)=-\chi_{s} \Delta \mathbf{n}(x, 0)$ and $\mathbf{J}(x, 0)=J_{s} \mathbf{n}(x, 0)$, constitute nine coupled first-order ordinary differential equations which can be integrated by a simple fourth-order Runge-Kutta scheme. Since this scheme does not yield a simple closed formula for the effective spin torque $\mathbf{T}$, however, we refrain from this approach here.

\section{CONCLUDING REMARKS}

In this paper, we have studied the spin torque due to an applied spin-polarized current entering the Landau-Lifshitz-Gilbert equation for the magnetization $-M_{s} \mathbf{n}(x, t)$ of a ferromagnetic metallic wire. Spin relaxation of the itinerant electrons has been included within a Bloch-Redfield formalism adapted to this problem. The equation of motion of the itinerant electrons can be iteratively solved within a gradient expansion scheme for current-induced spin torque $\mathbf{T}$ entering the LLG equation. The first order in this expansion contains only first-order derivative terms (in particular, $\partial_{x} \mathbf{n}$ ), and recovers the known 'adiabatic' result (11) and the 'nonadiabatic' correction (2). We prefer to call both terms 'adiabatic' here, since they are both of first order in the gradient expansion. The correction term discovered in Ref. 13] is in fact due to spin relaxation [13, 15, 16]. We have then explicitly derived all second-order contributions to the spin torque term. As they involve spatial and time derivatives of the magnetization, they generally induce a deformation of the magnetization profile as compared to the simple adiabatic shape. This has been illustrated for the example of a tail-to-tail transverse domain wall. Despite the fact that the DW gets distorted, it still moves uniformly with constant terminal velocity. We have shown that this velocity depends in a non-trivial way on the applied spin current density $J_{s}$. For small $J_{s}$, it essentially coincides with the known first-order result, which illustrates that the 'adiabatic' pinning of the DW is overcome by the $\beta$-term (2) in the spin torque. For larger spin currents, the DW velocity does not grow further with growing $J_{s}$ but instead diminishes again. Most likely, upon inclusion of even higher orders, this indicates a saturation of the DW velocity for large $J_{s}$. Importantly, a simple analytical expression for the leading non-adiabatic corrections to the known current-induced spin torque $\mathbf{T}_{a d}+\mathbf{T}_{Z L}$ has been provided in our work, see Eq. (32). We hope that this result will also find its significance in more elaborate micro-magnetic simulations. Finally, we remark that also the problem of pinning 8] of a DW by structural defects could be included in our formalism. The generalization to incorporate thermal ef- 
fects [46, 47] via a stochastic LLG equation appears possible as well.

\section{Acknowledgments}

We thank Gerrit Bauer, Rembert Duine, Mathias Kläui, and Gen Tatara for useful discussions. This work was supported by the SFB TR 12 of the DFG.

\section{APPENDIX A: RELAXATION KERNELS}

In this Appendix, we sketch the derivation of the relaxation kernels entering the equations of motion for the spin density and spin current density of the itinerant electrons. We use the abbreviations $\mathbf{K}_{s}(x) \equiv \mathbf{s}(x)$ and $\mathbf{K}_{J}(x) \equiv \mathbf{J}(x)$, and start from the master equation for the reduced density operator under the Markov approximation for our time-dependent system Hamiltonian $H_{S}(t)=H_{0}+H_{e x}(t)$. This master equation has been derived in detail in Ref. [30], and can be taken over directly. The derivation assumes that the system is linearly coupled to a collection of environmental harmonic oscillators (with 'bath' Hamiltonian $H_{B}$ and bath temperature $T$ ) via a bilinear system-bath coupling $H_{S B}$. Specifically, we take

$$
H_{S B}=\sum_{q=x, y, z ; \lambda=s, J} \int d x B_{\lambda, q}(x, t) K_{\lambda, q}(x)
$$

with fluctuating magnetic fields $B_{\mu, q}(x, t)$ produced by the harmonic bath. A key quantity is the (free) bath correlation function $\overline{B_{\mu, q}(x, t) B_{\nu, q^{\prime}}\left(x^{\prime}, t-\tau\right)}$, or its Fourier transform, the spectral density [32]. We will assume the spectral density to be real; neglecting its imaginary part only introduces small Lamb frequency shifts [28, 29, 30, 31] of no interest here. Thus we define

$$
\begin{aligned}
k_{q q^{\prime}, \mu \nu}\left(x, x^{\prime}, \omega\right) & =\frac{1}{2} \int_{-\infty}^{\infty} d \tau \cos (\omega \tau) \\
& \times \frac{B_{\mu, q}(x, t) B_{\nu, q^{\prime}}\left(x^{\prime}, t-\tau\right)}{}
\end{aligned}
$$

which is independent of $t$. For our purpose, it is sufficient to assume a spatially independent isotropic spectral density which yields an $x$-independent correlator $k_{q, \mu \nu}(\omega)=k_{q q^{\prime}, \mu \nu}\left(x, x^{\prime}, \omega\right) \delta_{q q^{\prime}}$. The coupling $H_{S B}$ is now assumed weak enough to justify a lowest-order perturbative scheme. Moreover, the bath autocorrelation time $\tau_{c}$ should be sufficiently short to justify the standard Markov (no memory) approximation. Our starting point is thus the Markovian master equation for the reduced density matrix $\rho(t)$ of the system [30],

$$
\frac{d \rho(t)}{d t}=-i\left[H_{S}(t), \rho(t)\right]-\frac{1}{2} \int_{-\infty}^{\infty} d \tau \overline{\left[H_{S B}(t),\left[e^{-i H_{S}(t) \tau} H_{S B}(t-\tau) e^{i H_{S}(t) \tau}, \rho(t)-\rho_{S}(t)\right]\right]},
$$

where the time-dependent Boltzmann density operator is given by

$$
\rho_{S}(t)=\frac{e^{-H_{S}(t) / k_{B} T}}{\operatorname{tr} e^{-H_{S}(t) / k_{B} T}}
$$

The system thus relaxes to the instantaneous Boltzmann distribution (A4) which contains the full time-dependent magnetic field generated by $\mathbf{n}(x, t)$ in $H_{e x}(t)$.

From this result, we can then derive the Bloch-Redfield equations, i.e., the equations of motion for the quantum statistical expectation values $\overline{\langle\mathbf{s}(x, t)\rangle}$ and $\overline{\langle\mathbf{J}(x, t)\rangle}$. (For notational simplicity, we omit the brackets and the overline $\overline{\langle\cdot\rangle}$ in most of the paper, but we always mean the quantum statistical expectation value. In this Appendix, we shall keep them for clarity.) Let us now consider the $r$-th component of $\overline{\left\langle\mathbf{K}_{\lambda}(x, t)\right\rangle}(\lambda=s, J)$, which obeys the equation of motion

$$
\frac{d}{d t} \overline{\left\langle K_{\lambda, r}(x, t)\right\rangle}=\sum_{\alpha \alpha^{\prime}} \frac{d \rho_{\alpha \alpha^{\prime}}(t)}{d t}\left\langle\alpha^{\prime}\left|K_{\lambda, r}(x, t)\right| \alpha\right\rangle,
$$

since there is no explicit time dependence present in the operator $\mathbf{K}_{\lambda}$. We have employed a suitable complete set of eigenstates, $H_{S}(t)|\alpha(t)\rangle=\alpha(t)|\alpha(t)\rangle$, where for simplicity $\alpha$ denotes both the quantum numbers and the eigenenergy, and $\sum_{\alpha}|\alpha\rangle\langle\alpha|=1$ (we keep the $t$ dependence of $\alpha$ implicit from now on). By inserting Eq. (A3) into Eq. (A5), we obtain first a part simply yielding the coherent terms in Eqs. (11) and (12). Here we focus on the relaxation part and discuss the term $\sim \rho(t)$ in Eq. A3 . The other term involving $\rho_{S}(t)$ follows from a simple substitution. Therefore, we have to calculate 


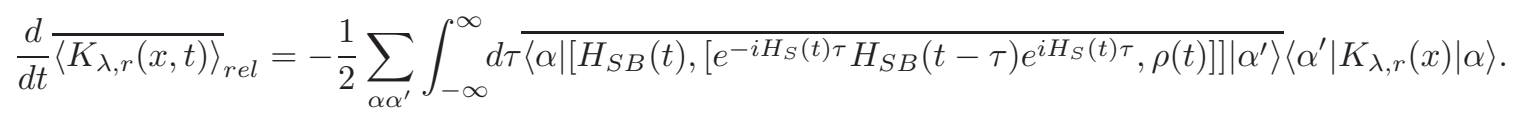

Expanding the double commutator yields four terms, of which one will be discussed in more detail. Inserting unities, we find such a term as

$$
\begin{aligned}
& \sum_{\alpha \alpha^{\prime} \beta \beta^{\prime}} \frac{1}{2} \int_{-\infty}^{\infty} d \tau \overline{\left\langle\alpha\left|H_{S B}(t)\right| \beta\right\rangle\left\langle\beta^{\prime}\left|H_{S B}(t-\tau)\right| \alpha^{\prime}\right\rangle} \\
& \times e^{i\left(\alpha^{\prime}-\beta^{\prime}\right) \tau}\left\langle\beta|\rho| \beta^{\prime}\right\rangle\left\langle\alpha^{\prime}\left|K_{\lambda, r}(x)\right| \alpha\right\rangle .
\end{aligned}
$$

Using the bath correlation functions introduced above, this can be written as

$$
\begin{aligned}
& \sum_{\alpha \beta q} \sum_{\mu \nu} \int d x^{\prime} d x^{\prime \prime}\left\langle\beta\left|K_{\nu, q}\left(x^{\prime \prime}\right)\right| \alpha\right\rangle \\
& \times\left\langle\alpha\left|K_{\lambda, r}(x) K_{\mu, q}\left(x^{\prime}\right) \rho\right| \beta\right\rangle k_{q, \mu \nu}(\beta-\alpha) .
\end{aligned}
$$

Collecting all four terms then yields the relaxation part

$$
\begin{aligned}
& \frac{d}{d t}{\overline{\left\langle K_{\lambda, r}(x)\right\rangle_{t, r e l}}}=\sum_{\alpha \beta q} \sum_{\mu \nu} \int d x^{\prime} d x^{\prime \prime}\left\langle\beta\left|K_{\nu, q}\left(x^{\prime \prime}\right)\right| \alpha\right\rangle \\
& \times\left\langle\alpha\left|\left[\left[K_{\lambda, r}(x), K_{\mu, q}\left(x^{\prime}\right)\right], \rho\right]\right| \beta\right\rangle k_{q, \mu \nu}(\beta-\alpha),(\mathrm{A} 7)
\end{aligned}
$$

where we exploit that $k_{q, \mu \nu}(\omega)$ is even in $\omega$.

Applying Eq. (A7) to the case $K_{s, r=z} \equiv s_{z}$ is straightforward upon using the Kac-Moody algebra and cyclic invariance of the trace. In the $q$-summation, the contribution for $q=x$ involves the matrix elements of the operator $s_{x}$. States $\alpha$ and $\beta$ joined by $s_{x}$ thus have $\beta-\alpha=\Delta$, the Larmor frequency for this problem. A similar reasoning applies to $q=y$, while $q=z$ does not contribute at all. Collecting all pieces yields for the relaxation part

$$
\frac{d}{d t}{\overline{\left\langle s_{z}(x, t)\right\rangle_{r e l}}}=-\frac{1}{\tau_{s, z}} \overline{\left\langle s_{z}(x, t)\right\rangle}
$$

with

$$
\frac{1}{\tau_{s, z}}=k_{x, s s}(\Delta)+k_{y, s s}(\Delta)+v^{2}\left[k_{x, J J}(\Delta)+k_{y, J J}(\Delta)\right]
$$

where we use $k_{q, \mu \nu}(\omega)=k_{q, \nu \mu}(\omega)$. For $r=x, y$, we proceed in the same way and find

$$
\frac{d}{d t}{\overline{\left\langle s_{x / y}(x, t)\right\rangle_{r e l}}}=-\frac{1}{\tau_{s, x / y}} \overline{\left\langle s_{x / y}(x, t)\right\rangle}
$$

with

$$
\frac{1}{\tau_{s, x / y}}=k_{y / x, s s}(\Delta)+k_{z, s s}(0)+v^{2}\left[k_{y / x, J J}(\Delta)+k_{z, J J}(0)\right] .
$$

Assuming isotropy, $\tau_{s, x} \approx \tau_{s, y} \approx \tau_{s, z} \approx \tau_{s}$, and adding the part from the stationary distribution (A4), we finally obtain the relaxation term (16) entering Eq. (11). In the same manner, $\boldsymbol{\Gamma}_{J}$ follows from such a calculation.
[1] I. Zutic, J. Fabian, and S. Das Sarma, Rev. Mod. Phys. 76, 323 (2004).

[2] Y. Tserkovnyak, A. Brataas, G.E.W. Bauer, and B.I. Halperin, Rev. Mod. Phys. 77, 1375 (2005).

[3] A. Brataas, G.E.W. Bauer, and P.J. Kelly, Phys. Rep. 427, 157 (2006).

[4] L. Berger, Phys. Rev. B 54, 9353 (1996).

[5] Ya.B. Bazaliy, B.A. Jones, and S.-C. Zhang, Phys. Rev. B 57, R3213 (1998).

[6] Z. Li and S. Zhang, Phys. Rev. B 70, 024417 (2004).

[7] J. Fernández-Rossier, M. Braun, A.S. Núnez, and A.H. MacDonald, Phys. Rev. B 69, 174412 (2004).

[8] G. Tatara and H. Kohno, Phys. Rev. Lett. 92, 086601 (2004); J. Shibata, G. Tatara, and H. Kohno, Phys. Rev. Lett. 94, 076601 (2005).

[9] F. Piéchon and A. Thiaville, Phys. Rev. B 75, 174414 (2007).

[10] For a review, see C.H. Marrows, Adv. Phys. 54, 585 (2005).

[11] I.N. Krivorotov, N.C. Emley, J.C. Sankey, S.I. Kiselev, D.C. Ralph, and R.A. Buhrman, Science 307, 228 (2005).
[12] J.Z. Sun, Phys. Rev. B 62, 570 (2000); A.A. Tulapurkar, Y. Suzuki, A. Fukushima, H. Kubota, H. Maehara, K. Tsunekawa, D.D. Djayaprawira, N. Watanabe, and S. Yuasa, Nature 438, 339 (2005).

[13] S. Zhang and Z. Li, Phys. Rev. Lett. 93, 127204 (2004).

[14] S.E. Barnes and S. Maekawa, Phys. Rev. Lett. 95, 107204 (2005).

[15] Y. Tserkovnyak, H. J. Skadsem, A. Brataas, and G.E.W. Bauer, Phys. Rev. B 74, 144405 (2006); H. J. Skadsem, Y. Tserkovnyak, A. Brataas, and G.E.W. Bauer, Phys. Rev. B 75, 094416 (2007).

[16] H. Kohno, G. Tatara, and J. Shibata, J. Phys. Soc. Jpn. 75, 113706 (2006).

[17] G. Tatara, T. Takayama, H. Kohno, J. Shibata, Y. Nakatani, and H. Fukuyama, J. Phys. Soc. Jpn. 75, 064708 (2006).

[18] R. A. Duine, A. S. Núñez, J. Sinova, and A. H. MacDonald, Phys. Rev. B 75, 214420 (2007).

[19] J. Xiao, A. Zangwill, and M.D. Stiles, Phys. Rev. B 73, 054428 (2006).

[20] G. Tatara, H. Kohno, J. Shibata, and K.-J. Lee, J. Phys. 
Soc. Jpn. 76, 054707 (2007)

[21] M.A. Ruderman and C. Kittel, Phys. Rev. 96, 99 (1954); T. Kasuya, Prog. Theor. Phys. 16, 45 (1956); K. Yosida, Phys. Rev. 106, 893 (1957); see also C. Kittel, Quantum Theory of Solids (Wiley, New York, 1987).

[22] E. Šimánek and B. Heinrich, Phys. Rev. B 67, 144418 (2003)

[23] V. K. Dugaev, V. R. Vieira, P. D. Sacramento, J. Barnaś, M. A. N. Araújo, and J. Berakdar, Phys. Rev. B 74, 054403 (2006).

[24] A.K. Nguyen, H. J. Skadsem, and A. Brataas, Phys. Rev. Lett. 98, 146602 (2007).

[25] V. Korenman, J.L. Murray, and R.E. Prange, Phys. Rev. B 16, 4032 (1977).

[26] K. Yosida, Theory of Magnetism, Springer Series in Solid State Sciences vol. 122, (Springer, Heidelberg, 1996).

[27] A. Vanhaverbeke and M. Viret, Phys. Rev. B 75, 024411 (2007).

[28] W.H. Louisell, Quantum Statistical Properties of Radiation (Wiley, New York, 1973).

[29] C.P. Slichter, Principles of Magnetic Resonance, 3rd ed. (Springer, Heidelberg, 1990).

[30] A. Abragam, The Principles of Nuclear Magnetism (Oxford University Press, London, 1961).

[31] V. May and O. Kühn, Charge and energy transfer dynamics in molecular systems (Wiley, Berlin, 2001).

[32] U. Weiss, Quantum Dissipative Systems, 2nd ed. (World Scientific, Singapore, 1998).

[33] G. Meier, M. Bolte, R. Eiselt, B. Krüger, D.-H. Kim, and P. Fischer, Phys. Rev. Lett. 98, 187202 (2007).
[34] M. Kläui, P.-O. Jubert, R. Allenspach, A. Bischof, J.A.C. Bland, G. Faini, U. Rüdiger, C.A.F. Vaz, L. Vila, and C. Vouille, Phys. Rev. Lett. 95, 026601 (2005).

[35] X. Waintal and M. Viret, Europhys. Lett. 65, 427 (2004).

[36] A. Thiaville, Y. Nakatani, J. Miltat, and Y. Suzuki, Europhys. Lett. 69, 990 (2005).

[37] J.I. Ohe and B. Kramer, Phys. Rev. Lett. 96, 027204 (2006).

[38] L. Balents and R. Egger, Phys. Rev. Lett. 85, 3464 (2000); Phys. Rev. B 64, 035310 (2001).

[39] A.O. Gogolin, A.A. Nersesyan, and A.M. Tsvelik, Bosonization and Strongly Correlated Systems (Cambridge University Press, 1998).

[40] M.A.N. Araujo, V.K. Dugaev, V.R. Vieira, J. Berakdar, and J. Barnas, Phys. Rev. B 74, 224429 (2006).

[41] H. Kohno and J. Shibata, J. Phys. Soc. Jpn. 76 , 063710 (2007).

[42] Y. Tserkovnyak, private communication.

[43] D. Y. Petrovykh, K. N. Altmann, H. Höchst, M. Laubscher, S. Maat, G. J. Mankey, and F. J. Himpsel, Appl. Phys. Lett. 73, 3459 (1998).

[44] S. Dubois, L. Piraux, J. M. George, K. Ounadjela, J. L. Duvail, A. Fert, Phys. Rev. B 60, 477 (1999).

[45] N. L. Schryer and L.R. Walker, J. Appl. Phys. 45, 5406 (1974).

[46] R. A. Duine, A. S. Núñez, and A. H. MacDonald, Phys. Rev. Lett. 98, 056605 (2007).

[47] M. Hatami, G. E. W. Bauer, Q. Zhang, and P. J. Kelly, Phys. Rev. Lett. 99, 066603 (2007). 